\title{
Channel Modelling and Performance Analysis of V2I Communication Systems in Blind Bend Scattering Environments
}

\author{
Ali Chelli ${ }^{1,}{ }^{*}$, Rami Hamdi ${ }^{2}$, and Mohamed-Slim Alouini ${ }^{1}$
}

\begin{abstract}
In this paper, we derive a new geometrical blind bend scattering model for vehicle-toinfrastructure (V2I) communications. The proposed model takes into account single-bounce and doublebounce scattering stemming from fixed scatterers located on both sides of a curved street. Starting from the geometrical blind bend model, the exact expression of the angle of departure (AOD) is derived. Based on this expression, the probability density function (PDF) of the AOD and the Doppler power spectrum are determined. Analytical expressions for the channel gain and the temporal autocorrelation function $(\mathrm{ACF})$ are provided under non-line-of-sight (NLOS) conditions. Additionally, we investigate the impact of the position of transmitting vehicle relatively to the receiving road-side unit on the channel statistics. Moreover, we study the performance of different digital modulations over a sum of singly and doubly scattered (SSDS) channel. Note that the proposed V2I channel model falls under the umbrella of SSDS channels since the transmitted signal undergoes a combination of single-bounce and double-bounce scattering. We study some characteristic quantities of SSDS channels and derive expressions for the average symbol error probability of several modulation schemes over SSDS channels with and without diversity combining. The validity of these analytical expressions is confirmed by computer-based simulations.
\end{abstract}

\section{INTRODUCTION}

In recent years, vehicle-to-infrastructure (V2I) communications and vehicle-to-vehicle (V2V) communications have attracted a lot of interest due to their numerous benefits. Using V2I and V2V communications, it is possible to reduce the number of road accidents and to improve traffic flow. The risk of accident increases if the driver does not have a full visibility of the road ahead. This situation occurs in a blind bend ${ }^{\dagger}$ environment as illustrated in Fig. 1. In this case, V2I communications can provide the driver with necessary information concerning the existence of potential hazards few meters ahead or with instructions to drive more carefully to avoid a crash with vehicles approaching from the opposite direction. For the development of such V2I safety applications, the exact knowledge of the V2I fading channel statistics is necessary.

V2I communications fall under the category of mobile-to-fixed communications which comprises as well the classical cellular channel. Unfortunately, the channel models of cellular systems cannot be used to describe the fading behaviour of V2I channels. In fact, V2I environments are characterized by a higher mobility compared to cellular environments. Additionally, existing cellular channel models such as the COST-Hata model [1] have been validated for the case where the base station height is larger than $30 \mathrm{~m}$ and the distance between the base station and the receiver is above $1 \mathrm{~km}$. By contrast, for

\footnotetext{
Received 25 July 2013, Accepted 12 December 2013, Scheduled 16 December 2013

* Corresponding author: Ali Chelli (ali.chelli@kaust.edu.sa).

1 Computer, Electrical, and Mathematical Science and Engineering (CEMSE) Division, King Abdullah University of Science and Technology (KAUST), Thuwal, Makkah Province, Kingdom of Saudi Arabia. ${ }^{2}$ Department of Electrical Engineering, Qatar University, Doha, Qatar. Ali Chelli and Mohamed-Slim Alouini are members of the KAUST Strategic Research Initiative on Uncertainty Quantification in Science and Engineering.

$\dagger$ The term blind bend is utilized to refer to the environment where the street curvature do not allow the driver to have a full visibility of the road ahead.
} 
V2I communications, the height of the road-side unit (RSU) is typically between 1 to $6 \mathrm{~m}$ and the V2I communication range is less than few hundred meters [2]. The high mobility in vehicular environments combined with the differences in antenna height and coverage range between V2I channels and cellular channels make the transmitted waves experience different propagation mechanisms. These dissimilarities have a major impact on the channel statistics, such as the Doppler power spectral density (PSD), the Doppler spread, the power delay profile, and the delay spread. Hence, the channel models of cellular systems cannot be used to model V2I channels.

Most of the existing work on V2I communications focuses on performance analysis of V2I systems based on field testing. In [3], a field test campaign has been conducted to evaluate the packet delivery rate for different streets' layout and different traffic conditions. It turned out that all these factors have an impact on V2I system performance. In [4], the frame success ratio and the goodput for V2I communications in a tunnel are assessed. Whereas in [2], the main focus is the modelling of the path loss for V2I channels. The obtained path loss model can be utilized to optimize the deployment of RSUs. However, a path loss model is not enough to describe the fading behaviour of the channel. To get a complete picture of the channel variations, we need to model as well V2I small scale fading. Small scale fading models allow obtaining the statistical properties of the received signal envelope. This information is useful for the performance evaluation and the optimization of different transmission schemes over V2I channels. To the best of our knowledge, no V2I channel model for small scale fading can be found in the literature. To bridge this gap, we propose a novel V2I channel model for small scale fading.

In this work, we use a geometry-based approach to derive a narrowband single-input single-output (SISO) V2I channel model for blind bend environments. The geometry-based approach has been used in [5] to develop a three dimensional channel model for cellular propagation environments. Two dimensional geometry-based channel model for cellular environments are proposed in [6,7]. Starting from the geometrical blind bend model, we derive the exact expression for the angles of departure (AOD). Based on this expression, the probability density function (PDF) of the AOD and the PSD of the Doppler frequency are derived. The proposed channel model takes into account single-bounce (SB) and double-bounce (DB) scattering ${ }^{\ddagger}$ form fixed scatterers located on both sides of the road. We assume that the propagation occurs under non-line-of-sight (NLOS) conditions. In a real-world propagation scenario, it is unrealistic to assume that the received signal would be a result of a pure SB or pureDB scattering mechanism [8]. In general, a transmitted waveform experiences both SB and DB scattering. This fact has been confirmed by channel measurement in vehicular environments $[9,10]$. Therefore, the proposed channel model takes into account SB and DB scattering.

The proposed channel model is useful for the design and analysis of future V2I communication systems. More specifically, using the probability density function (PDF) of the fading amplitude we can evaluate the performance of various digital modulations over V2I channels. The investigated V2I channel falls under the umbrella of $\operatorname{SSDS}^{\S}$ channels [8]. For SSDS channels, the received signal is a superposition of SB and DB scattering components as it is the case of our V2I channel model. It is worth mentioning that the SSDS channel comprises the Rayleigh channel and the double Rayleigh channel as special cases which make the SSDS channel more general. If we have pure SB (DB) scattering the received signal amplitude follows a Rayleigh (double Rayleigh) distribution.

The main contributions of this paper compared to our work in [11] consist of the study of some characteristic quantities of the SSDS channel as well as the performance evaluation of various modulation schemes over SSDS channels. We show in this paper that the severity of fading for SSDS channels is higher compared to Rayleigh channels. Besides, we investigate the characteristic quantities of SSDS channels, such as the PDF of the signal-to-noise ratio (SNR), the outage probability, and the moment generating function (MGF) of the SNR. Using an MGF-based approach, we derive expressions for the average symbol error probability (SEP) of M-ary phase shift keying (M-PSK) and M-ary quadrature amplitude modulation (M-QAM) modulation schemes without diversity, with maximal ratio combining (MRC), and with selective combining (SC).

The remainder of the paper is organized as follows. In Section 2, we present the geometrical blind bend model and derive an expression for the AOD. Based on this geometrical model, we derive a reference

\footnotetext{
¥ Strictly speaking, we have to distinguish between reflection and scattering, as they are different propagation mechanisms. In this paper, however, we will utilize the expression "scattering" and "scatterers" to comprise both of those effects.

$\S$ SSDS stands for sum of singly and doubly scattered.
} 
model in Section 3. Section 4 focuses on the statistical characterization of the proposed channel model. Analytical expressions are provided for the PDF of the AOD, the temporal autocorrelation function $(\mathrm{ACF})$, and the Doppler PSD. Additionally, these statistical properties are evaluated numerically and validated by simulations. The performance evaluation of various transmission schemes over SSDS channels is performed in Section 5. Finally, we draw the conclusion in Section 6.

\section{THE GEOMETRICAL BLIND BEND MODEL}

A blind bend propagation environment for V2I communications is presented in Fig. 1. For this scenario, a vehicle denoted by $\mathrm{MS}^{\|}$and moving in a curved street as shown in Fig. 1. Due to the curvature of the street, the driver is not able to see the road ahead. The transmitter MS tries to communicate with a fixed RSU deployed on the other side of the road as illustrated in Fig. 1. The RSU, which plays the role of a fixed receiver, can provide the driver with information pertaining to the existence of hazards on the road ahead as well as the traffic and road conditions. Our objective is to develop an appropriate model for the V2I channel between the transmitting vehicle MS and the fixed receiver referred to as RSU. Towards this aim, we first need to produce a representative geometrical model for the propagation scenario presented in Fig. 1. The geometrical model can be obtained by replacing the fixed scattering objects (buildings and trees) located on both sides of the street with scatterers located on a curved line having the same shape as the street boundaries. The obtained geometrical model is illustrated in Fig. 2.

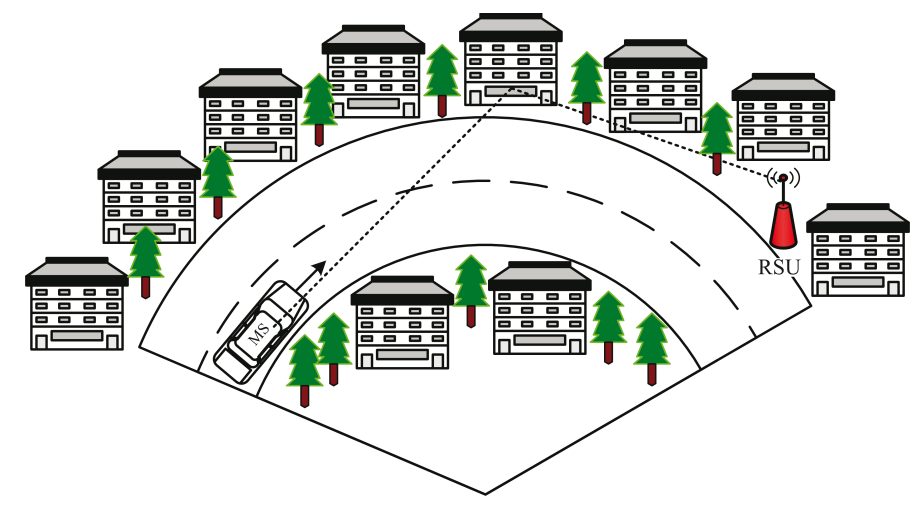

Figure 1. A blind bend propagation environment for V2I communications.

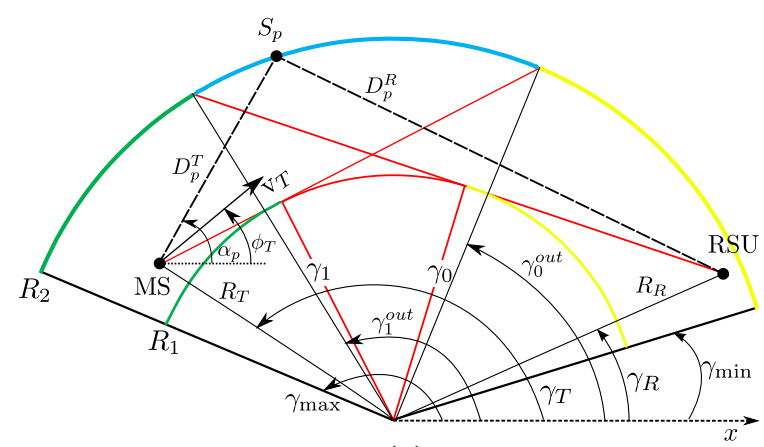

(a)

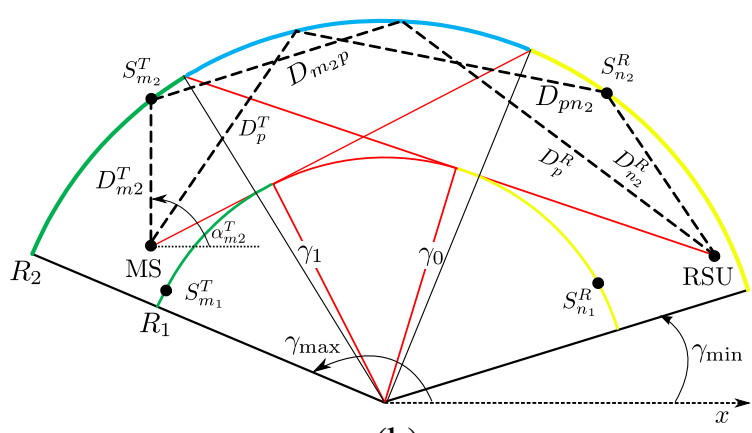

(b)

Figure 2. The geometrical blind bend model illustrating (a) the SB and (b) the DB scattering mechanisms.

We assume that we have NLOS propagation conditions. The location of the transmitter MS and the receiver RSU are given by their polar coordinates $\left(R_{T}, \gamma_{T}\right)$ and $\left(R_{R}, \gamma_{R}\right)$. The transmitter MS is

\| The term MS stands for mobile station. 
moving with a speed $\mathrm{v}_{T}$ in the direction determined by the angle $\phi_{T}$. The angle $\phi_{T}$ can be expressed in terms $\gamma_{T}$ as follows

$$
\phi_{T}=\gamma_{T}-\frac{\pi}{2}
$$

The road boundaries are delimited by an inner circle-sector of radius $R_{1}$ and an outer circle-sector of radius $R_{2}$. The angle of scatterer (AOS) $\gamma$ is confined to the interval $\left[\gamma_{\min }, \gamma_{\max }\right]$, where $\gamma_{\min }$ and $\gamma_{\max }$ are constant parameters that can be modified depending on the street layout. The proposed V2I model takes into account SB and DB scattering form fixed scatterers located on the inner and outer circle-sectors.

Our first task is to determine the scatterers that contribute to SB scattering. To this end, we have to draw the tangent segments to the inner circle passing through the transmitter and the receiver. We find out that the scatterers $S_{p}(p=1, \ldots, P)$ located on the blue arc [see Fig. 2] of the outer circle are the only scatterers contributing to SB scattering. The edges of this blue arc correspond to the intersection of the outer circle and the tangent segment to the inner circle passing through the transmitter and the receiver. For SB scattering, the plane wave emitted from the transmitter travels over the scatterer $S_{p}$ located on the blue arc before reaching the receiver directly without any further scattering. The AOD and the AOS associated with the scatterer $S_{p}$ are referred to as $\alpha_{p}$ and $\gamma_{p}$, respectively. Note that the $\operatorname{AOS} \gamma_{p}$ is within the interval $\left[\gamma_{0}^{\text {out }}, \gamma_{1}^{\text {out }}\right]$ [see Fig. 2], where

$$
\begin{aligned}
& \gamma_{0}^{\text {out }}=\gamma_{T}-\arccos \left(\frac{R_{1}}{R_{T}}\right)-\arccos \left(\frac{R_{1}}{R_{2}}\right) \\
& \gamma_{1}^{\text {out }}=\gamma_{R}+\arccos \left(\frac{R_{1}}{R_{R}}\right)+\arccos \left(\frac{R_{1}}{R_{2}}\right) .
\end{aligned}
$$

For the scatterers belonging to the red arc on the inner circle [see Fig. 2], no ray emitted from the transmitter can reach these scatterers directly and no ray stemming from these scatterers can reach the receiver directly. Hence, these scatterers only contribute to triple-bounce scattering only. The edges of the red arc correspond to the intersection of the inner circle and the tangent segments to the inner circle passing through the transmitter and the receiver. For the scatterers located on the red arc, the AOS is confined to the interval $\left[\gamma_{0}, \gamma_{1}\right]$ [see Fig. 2], where

$$
\begin{aligned}
& \gamma_{0}=\gamma_{R}+\arccos \left(\frac{R_{1}}{R_{R}}\right) \\
& \gamma_{1}=\gamma_{T}-\arccos \left(\frac{R_{1}}{R_{T}}\right) .
\end{aligned}
$$

In our model, we do not take into account triple-bounce scattering. It is well known that the energy absorbed during the scattering process increases as the order of scattering increases. Thus, SB and DB scattering components bear more energy than triple-bounce components. Hence, by neglecting triple-bounce components, we simplify the channel model without reducing much its accuracy. It is worth mentioning that most of the existing geometry-based channel models [9,10,12-14] for vehicular environments do not take into consideration triple-bounce scattering since it has much less energy relatively to SB and DB scattering.

The DB scattering component are composed of: (i) waves scattered first by the scatterers around the transmitter located on the green arc (denoted as $S_{m_{1}}^{T}$ and $S_{m_{2}}^{T}$ ) then by the scatterers $S_{p}$ belonging to the blue arc to finally reach the receiver, (ii) waves scattered first by the scatterers $S_{p}$ and then by the scatterers around the receiver located on the yellow arc (referred to as $S_{n_{1}}^{R}$ and $S_{n_{2}}^{R}$ ) before reaching the receiver. It has to be noted that the AOS for the scatterers $S_{m_{1}}^{T}\left(m_{1}=1, \ldots, M_{1}\right)$ (located on the green arc on the inner circle) belong to the interval $\left[\gamma_{1}, \gamma_{\max }\right]$, whereas for the scatterers $S_{m_{2}}^{T}\left(m_{2}=1, \ldots, M_{2}\right)$ (located on the green arc on the outer circle) are within the interval $\left[\gamma_{1}^{\text {out }}, \gamma_{\max }\right]$. For the scatterers around the receiver that contribute toDB scattering, they are denoted as $S_{n_{1}}^{R}\left(n_{1}=1, \ldots, N_{1}\right)$ and $S_{n_{2}}^{R}\left(n_{2}=1, \ldots, N_{2}\right)$ and are located on the yellow arc on the inner and outer circle, respectively. The 
AOS are confined to the interval $\left[\gamma_{\min }, \gamma_{0}\right]$ for the scatterers $S_{n_{1}}^{R}$. For the scatterers $S_{n_{2}}^{R}$, the AOS is within the interval $\left[\gamma_{\min }, \gamma_{0}^{\text {out }}\right]$. Since we are investigating a V2I channel model, the statistics of the channel, such the Doppler PSD and the temporal ACF, depend only on the AOD. It can be shown using Fig. 2 and some trigonometric identities that the AOD $\alpha_{p}$ associated with the scatterer $S_{p}$ can be expressed as

$$
\alpha_{p}=f\left(\gamma_{p}\right)= \begin{cases}f_{1}\left(\gamma_{p}\right)+\pi & \text { if } \pi-\arccos \left(-\frac{R_{T} \cdot \cos \left(\gamma_{T}\right)}{R_{2}}\right) \leq \gamma_{p} \leq \pi \\ f_{1}\left(\gamma_{p}\right) & \text { if } 0 \leq \gamma_{p}<\pi-\arccos \left(-\frac{R_{T} \cdot \cos \left(\gamma_{T}\right)}{R_{2}}\right)\end{cases}
$$

where $\gamma_{p}$ is the AOS of the scatterer $S_{p}$ and

$$
f_{1}\left(\gamma_{p}\right)=\arctan \left(\frac{R_{2} \cdot \sin \left(\gamma_{p}\right)-R_{T} \cdot \sin \left(\gamma_{T}\right)}{R_{2} \cdot \cos \left(\gamma_{p}\right)-R_{T} \cdot \cos \left(\gamma_{T}\right)}\right) .
$$

Similarly, the AOD $\alpha_{m_{2}}^{T}$ associated with the scatterer $S_{m_{2}}^{T}$ can be written as $\alpha_{m_{2}}^{T}=f\left(\gamma_{m_{2}}^{T}\right)$, with $\gamma_{m_{2}}^{T}$ the AOS associated with the scatterer $S_{m_{2}}^{T}$.

The AOD $\alpha_{m_{1}}^{T}$ related to the scatterer $S_{m_{1}}^{T}$ can be obtained as

$$
\alpha_{m_{1}}^{T}=g\left(\gamma_{m_{1}}^{T}\right)= \begin{cases}g_{1}\left(\gamma_{m_{1}}^{T}\right)+\pi & \text { if } \pi-\arccos \left(-\frac{R_{T} \cdot \cos \left(\gamma_{T}\right)}{R_{1}}\right) \leq \gamma_{m_{1}}^{T} \leq \pi \\ g_{1}\left(\gamma_{m_{1}}^{T}\right) & \text { if } 0 \leq \gamma_{m_{1}}^{T}<\pi-\arccos \left(-\frac{R_{T} \cdot \cos \left(\gamma_{T}\right)}{R_{1}}\right),\end{cases}
$$

where $\gamma_{m_{1}}^{T}$ refers to the AOS of the scatterer $S_{m_{1}}^{T}$ and

$$
g_{1}\left(\gamma_{m_{1}}^{T}\right)=\arctan \left(\frac{R_{1} \cdot \sin \left(\gamma_{m_{1}}^{T}\right)-R_{T} \cdot \sin \left(\gamma_{T}\right)}{R_{1} \cdot \cos \left(\gamma_{m_{1}}^{T}\right)-R_{T} \cdot \cos \left(\gamma_{T}\right)}\right)
$$

\section{REFERENCE MODEL}

Starting from the geometrical model illustrated in Fig. 2, we derive in this section a reference model for the SISO V2I channel. The complex channel gain describing the link between the transmitter and the receiver can be expressed as

$$
g(t)=g^{\mathrm{SB}}(t)+g^{\mathrm{DB}}(t) .
$$

The channel gains caused by $\mathrm{SB}$ and $\mathrm{DB}$ scattering are referred to as $g^{\mathrm{SB}}(t)$ and $g^{\mathrm{DB}}(t)$, respectively. In the following, we derive analytical expressions for these channel gains.

\subsection{SB Component}

The $p$ th plane wave emitted from the transmitter MS travels over the scatterer $S_{p}(p=1, \ldots, P)$ before reaching the receiver RSU. For the reference model, we consider an infinite number of scatterers, i.e., $P \rightarrow \infty$. The channel gain pertaining to SB scattering $g^{\mathrm{SB}}(t)$ can be written as

$$
g^{\mathrm{SB}}(t)=\lim _{P \rightarrow \infty} \sqrt{\frac{\eta_{\mathrm{SB}}}{P}} \sum_{p=1}^{P} c_{p} e^{j\left(2 \pi f_{p} t+\theta_{p}\right)},
$$

where

$$
\begin{aligned}
& c_{p}=e^{-j \frac{2 \pi}{\lambda}\left(D_{p}^{T}+D_{p}^{R}\right)} \\
& f_{p}=f_{\max } \cos \left(\alpha_{p}-\phi_{T}\right) .
\end{aligned}
$$


The terms $f_{p}$ and $f_{\max }=\mathrm{v}_{T} / \lambda$ represent the Doppler frequency and the maximum Doppler frequency due to the movement of the transmitter, respectively, and $\lambda$ stands for the wavelength. The quantity $c_{p}$ accounts for the phase shift associated with the total distance travelled by the $p$ th plane wave. The term $D_{p}^{T}\left(D_{p}^{R}\right)$ denotes the distance between the scatterer $S_{p}$ and the transmitter (the receiver). The mean power of the SB component is referred to as $\eta_{\mathrm{SB}}$. The scatterer $S_{p}$ introduces a random phase shift $\theta_{p}$ having a uniform distribution over the interval $[0,2 \pi)$. Note that the phase shifts $\theta_{p}(p=1, \ldots, P)$ are independent identically distributed (i.i.d.) random variables (RVs).

\subsection{DB Component}

the DB component $g^{\mathrm{DB}}(t)$ is a superposition of two terms $g^{\mathrm{DBT}}(t)$ and $g^{\mathrm{DBR}}(t)$. The channel gain $g^{\mathrm{DBT}}(t)$ is caused byDB scattering from the scatterers around the transmitter $\left(S_{m_{1}}^{T}\right.$ or $\left.S_{m_{2}}^{T}\right)$ and the scatterers $S_{p}$. This channel gain can be expressed as

$$
g^{\mathrm{DBT}}(t)=\sum_{i=1}^{2} w_{i, T} \lim _{M_{i}, P \rightarrow \infty} \sqrt{\frac{\eta_{\mathrm{DBT}}}{M_{i} P}} \sum_{m_{i}, p=1}^{M_{i}, P} c_{m_{i} p} e^{j\left(2 \pi f_{m_{i}}^{T} t+\theta_{m_{i} p}\right)},
$$

where

$$
\begin{aligned}
c_{m_{i} p} & =e^{-j \frac{2 \pi}{\lambda}\left(D_{m_{i}}^{T}+D_{m_{i} p}+D_{p}^{R}\right)} \\
f_{m_{i}}^{T} & =f_{\max } \cos \left(\alpha_{m_{i}}^{T}-\phi_{T}\right) \\
\theta_{m_{i} p} & =\left(\theta_{m_{i}}+\theta_{p}\right) \bmod 2 \pi .
\end{aligned}
$$

The terms $w_{i, T}$ are weighting factors. To ensure that the mean power of $g^{\mathrm{DBT}}(t)$ is equal to $\eta_{\mathrm{DBT}}$, we impose the boundary condition $\sum_{i=1}^{2} w_{i, T}^{2}=1$. The quantity $c_{m_{i} p}$ represents the phase shift associated with the total distance travelled from the transmitter to the receiver via the scatterers $S_{m_{i}}^{T}(i=1,2)$ and $S_{p}$. The distances between the transmitter and the scatterer $S_{m_{i}}^{T}(i=1,2)$ is denoted by $D_{m_{i}}^{T}$. The distance between the scatterer $S_{p}$ and the receiver (the scatterer $S_{m_{i}}^{T}$ ) is referred to as $D_{p}^{R}\left(D_{m_{i} p}\right)$. The terms $\theta_{m_{i}}$ and $\theta_{p}$ are the phase shifts stemming from the scatterers $S_{m_{i}}^{T}$ and $S_{p}$, respectively. It is worth mentioning that $\theta_{m_{i}}, \theta_{m_{i}}$, and $\theta_{p}$ are i.i.d. RVs uniformly distributed over $[0,2 \pi)$. In (12c), the notation mod stands for the modulo operation.

The channel gain $g^{\mathrm{DBR}}(t)$ is associated with the waves that are first scattered by the scatterers $S_{p}$ and then by the scatterers $S_{n_{i}}^{R}(i=1,2)$ before reaching the receiver. This channel gain can be written as

$$
g^{\mathrm{DBR}}(t)=\sum_{i=1}^{2} w_{i, R} \lim _{P, N_{i} \rightarrow \infty} \sqrt{\frac{\eta_{\mathrm{DBR}}}{P N_{i}}} \sum_{p, n_{i}=1}^{P, N_{i}} c_{p n_{i}} e^{j\left(2 \pi f_{p} t+\theta_{p n_{i}}\right)},
$$

where

$$
\begin{aligned}
c_{p n_{i}} & =e^{-j \frac{2 \pi}{\lambda}\left(D_{p}^{T}+D_{p n_{i}}+D_{n_{i}}^{R}\right)} \\
f_{p} & =f_{\max } \cos \left(\alpha_{p}-\phi_{T}\right) \\
\theta_{p n_{i}} & =\left(\theta_{p}+\theta_{n_{i}}\right) \quad \bmod 2 \pi
\end{aligned}
$$

The terms $w_{i, R}$ are weighting factors. To ensure that the mean power of $g^{\mathrm{DBR}}(t)$ is equal to $\eta_{\mathrm{DBR}}$, we impose the boundary condition $\sum_{i=1}^{2} w_{i, R}^{2}=1$. The quantity $c_{p n_{i}}$ is the phase shift associated with the total distance travelled from the transmitter to the receiver via the scatterers $S_{p}$ and $S_{n_{i}}^{R}(i=1,2)$. The terms $\theta_{n_{i}}$ and $\theta_{p}$ are the phase shifts due to the scatterers $S_{n_{i}}^{R}$ and $S_{p}$, respectively. Note that $\theta_{p n_{i}}, \theta_{p}$, and $\theta_{n_{i}}$ are i.i.d. RVs uniformly distributed over $[0,2 \pi)$. 


\section{STATISTICAL CHARACTERIZATION}

This section is devoted to the study of the statistical properties of the proposed blind bend channel model. Analytical expressions are derived for the PDF of the AOD, the temporal ACF, and the Doppler PSD.

\subsection{PDF of the AOD}

In this section, we compute the PDF of the AODs $\alpha_{p}, \alpha_{m_{2}}^{T}$, and $\alpha_{m_{1}}^{T}$. We start by deriving the PDF of the AOD $\alpha_{p}$. The AOS $\gamma_{p}$ associated with the scatterer $S_{p}$ are uniformly distributed over the interval $\left[\gamma_{0}^{\text {out }}, \gamma_{1}^{\text {out }}\right]$. Thus, the PDF of the AOS $\gamma_{p}$ can be written as

$$
p_{\gamma_{p}}\left(\gamma_{p}\right)= \begin{cases}\frac{1}{\Delta \gamma_{p}} & \text { if } \gamma_{0}^{\text {out }} \leq \gamma_{p} \leq \gamma_{1}^{\text {out }} \\ 0 & \text { otherwise }\end{cases}
$$

where $\Delta \gamma_{p}=\gamma_{1}^{\text {out }}-\gamma_{0}^{\text {out }}$. Based on the relationship (4) between the AOD $\alpha_{p}$ and the AOS $\gamma_{p}$ and using the transformation of RVs fundamental theorem [15], we can obtain the the PDF of the AOD $\alpha_{p}$ as follows

$$
p_{\alpha_{p}}\left(\alpha_{p}\right)= \begin{cases}\frac{2}{\Delta \gamma_{p}} \frac{1}{1+\frac{R_{2}^{2}-R_{T}^{2}}{h\left(\alpha_{p}, R_{2}\right)}} & \text { if } \alpha_{0}^{\text {out }} \leq \alpha_{p} \leq \alpha_{1}^{\text {out }} \\ 0 & \text { otherwise }\end{cases}
$$

where $\alpha_{0}^{\text {out }}=f\left(\gamma_{0}^{\text {out }}\right), \alpha_{1}^{\text {out }}=f\left(\gamma_{1}^{\text {out }}\right)$ and

$$
h\left(\alpha_{p}, R_{2}\right)=R_{2}^{2}+R_{T}^{2}-2 R_{T} R_{2} \cdot \cos \left(\arcsin \left(\frac{R_{T} \cdot \sin \left(\gamma_{T}-\alpha_{p}\right)}{R_{2}}\right)+\alpha_{p}-\gamma_{T}\right) .
$$

The AOS $\gamma_{m_{2}}^{T}$ are uniformly distributed over $\left[\gamma_{1}^{\text {out }}, \gamma_{\max }\right]$. Using the transformation of RVs, the $\mathrm{PDF}$ of the $\mathrm{AOD} \alpha_{m_{2}}^{T}=f\left(\gamma_{m_{2}}^{T}\right)$ can be expressed as

$$
p_{\alpha_{m_{2}}^{T}}\left(\alpha_{m_{2}}^{T}\right)= \begin{cases}\frac{2}{\Delta \gamma_{m_{2}}^{T}} \frac{1}{1+\frac{R_{2}^{2}-R_{T}^{2}}{h\left(\alpha_{m_{2}}^{T}, R_{2}\right)}} & \text { if } \alpha_{1}^{\text {out }} \leq \alpha_{m_{2}}^{T} \leq \alpha_{\max } \\ 0 & \text { otherwise }\end{cases}
$$

where $\Delta \gamma_{m_{2}}^{T}=\gamma_{\max }-\gamma_{1}^{\text {out }}$ and $\alpha_{\max }=f\left(\gamma_{\max }\right)$.

The AOS $\gamma_{m_{1}}^{T}$ are uniformly distributed over $\left[\gamma_{1}, \gamma_{\max }\right]$. Using the relationship (6) between the AOS $\gamma_{m_{1}}^{T}$ and the AOD $\alpha_{m_{1}}^{T}$, the PDF of the AOD $\alpha_{m_{1}}^{T}$ can be determined as

$$
p_{\alpha_{m_{1}}^{T}}\left(\alpha_{m_{1}}^{T}\right)= \begin{cases}\frac{2}{\Delta \gamma_{m_{1}}^{T}} \frac{1}{1+\frac{R_{1}^{2}-R_{T}^{2}}{h\left(\alpha_{m_{1}}^{T}, R_{1}\right)}} & \text { if } \alpha_{\min } \leq \alpha_{m_{2}}^{T} \leq \alpha_{1} \\ 0 & \text { otherwise, }\end{cases}
$$

where $\Delta \gamma_{m_{1}}^{T}=\gamma_{\max }-\gamma_{1}, \alpha_{1}=g\left(\gamma_{1}\right)$, and $\alpha_{\min }=g\left(\gamma_{\max }\right)$.

\subsection{Temporal ACF}

In this section, we derive the temporal ACF of the proposed SISO V2I channel model. This function is defined as

$$
\begin{aligned}
R(\tau)= & E\left\{g^{*}(t) g(t+\tau)\right\}=E\left\{\left(g^{\mathrm{SB}}(t)\right)^{*} g^{\mathrm{SB}}(t+\tau)\right\}+E\left\{\left(g^{\mathrm{DBT}}(t)\right)^{*} g^{\mathrm{DBT}}(t+\tau)\right\} \\
& +E\left\{\left(g^{\mathrm{DBR}}(t)\right)^{*} g^{\mathrm{DBR}}(t+\tau)\right\}=R^{\mathrm{SB}}(\tau)+R^{\mathrm{DBT}}(\tau)+R^{\mathrm{DBR}}(\tau),
\end{aligned}
$$


where $(\cdot)^{*}$ denotes the complex conjugate, and $E\{\cdot\}$ stands for the expectation operator. The temporal $\mathrm{ACF}$ due to SB scattering can be determined as

$$
\begin{aligned}
R^{\mathrm{SB}}(\tau) & =E\left\{\left(g^{\mathrm{SB}}(t)\right)^{*} g^{\mathrm{SB}}(t+\tau)\right\}=\lim _{P \rightarrow \infty} \frac{\eta_{\mathrm{SB}}}{P} \sum_{p=1}^{P} E\left\{e^{j\left(2 \pi f_{p} \tau\right)}\right\} \\
& =\eta_{\mathrm{SB}} \int_{\alpha_{0}^{\text {out }}}^{\alpha_{1}^{\text {out }}} e^{j\left(2 \pi f_{p}\left(\alpha_{p}\right) \tau\right)} p_{\alpha_{p}}\left(\alpha_{p}\right) d \alpha_{p} .
\end{aligned}
$$

In (21), the expectation operator is applied to the RV $\alpha_{p}$ which allows obtaining the result in (22). Note that $f_{p}$ depends on $\alpha_{p}$ according to (10b). The temporal ACF associated with the channel gain $g^{\mathrm{DBT}}(t)$ can be written as

$$
\begin{aligned}
R^{\mathrm{DBT}}(\tau) & =E\left\{\left(g^{\mathrm{DBT}}(t)\right)^{*} g^{\mathrm{DBT}}(t+\tau)\right\}=\sum_{i=1}^{2} w_{i, T}^{2} \lim _{M_{i}, P \rightarrow \infty} \frac{\eta_{\mathrm{DBT}}}{M_{i} P} \sum_{m_{i}=1}^{M_{i}} P E\left\{e^{j\left(2 \pi f_{m_{i}}^{T} \tau\right)}\right\} \\
& =w_{1, T}^{2} \eta_{\mathrm{DBT}} \int_{\alpha_{\min }}^{\alpha_{1}} e^{j\left(2 \pi f_{m_{1}}^{T}\left(\alpha_{m_{1}}^{T}\right) \tau\right)} p_{\alpha_{m_{1}}^{T}}\left(\alpha_{m_{1}}^{T}\right) d \alpha_{m_{1}}^{T}+w_{2, T}^{2} \eta_{\mathrm{DBT}} \int_{\alpha_{1}^{\text {out }}}^{\alpha_{\max }} e^{j\left(2 \pi f_{m_{2}}^{T}\left(\alpha_{m_{2}}^{T}\right) \tau\right)} p_{\alpha_{m_{2}}^{T}}\left(\alpha_{m_{2}}^{T}\right) d \alpha_{m_{2}}^{T} .
\end{aligned}
$$

The temporal ACF $R^{\mathrm{DBR}}(\tau)$ can be expressed as

$$
\begin{aligned}
R^{\mathrm{DBR}}(\tau) & =E\left\{\left(g^{\mathrm{DBR}}(t)\right)^{*} g^{\mathrm{DBR}}(t+\tau)\right\}=\sum_{i=1}^{2} w_{i, R}^{2} \lim _{N_{i}, P \rightarrow \infty} \frac{\eta_{\mathrm{DBR}}}{N_{i} P} \sum_{p=1}^{P} N_{i} E\left\{e^{j\left(2 \pi f_{p} \tau\right)}\right\} \\
& =\sum_{i=1}^{2} w_{i, R}^{2} \eta_{\mathrm{DBR}} \int_{\alpha_{0}^{\text {out }}}^{\alpha_{1}^{\text {out }}} e^{j\left(2 \pi f_{p}\left(\alpha_{p}\right) \tau\right)} p_{\alpha_{p}}\left(\alpha_{p}\right) d \alpha_{p}=\eta_{\mathrm{DBR}} \int_{\alpha_{0}^{\text {out }}}^{\alpha_{1}^{\text {out }}} e^{j\left(2 \pi f_{p}\left(\alpha_{p}\right) \tau\right)} p_{\alpha_{p}}\left(\alpha_{p}\right) d \alpha_{p} .
\end{aligned}
$$

\subsection{Doppler PSD}

This section focus on the investigation of the Doppler PSD. It is well known that the Doppler PSD is obtained by applying the Fourier transform on the the temporal ACF. Thus, we can write the Doppler PSD of the V2I channel as

$$
S_{g}(f)=\mathcal{F}[R(\tau)]=\mathcal{F}\left[R^{\mathrm{SB}}(\tau)\right]+\mathcal{F}\left[R^{\mathrm{DBT}}(\tau)\right]+\mathcal{F}\left[R^{\mathrm{DBR}}(\tau)\right]=S^{\mathrm{SB}}(f)+S^{\mathrm{DBT}}(f)+S^{\mathrm{DBR}}(f),
$$

where $\mathcal{F}[\cdot]$ denotes the Fourier transform and

$$
\begin{aligned}
S^{\mathrm{SB}}(f) & =\eta_{\mathrm{SB}} p_{f_{p}}(f) \\
S^{\mathrm{DBR}}(f) & =\eta_{\mathrm{DBR}} p_{f_{p}}(f) \\
S^{\mathrm{DBT}}(f) & =\eta_{\mathrm{DBT}}\left(w_{1, T}^{2} p_{f_{m_{1}}^{T}}(f)+w_{2, T}^{2} p_{f_{m_{2}}^{T}}(f)\right) .
\end{aligned}
$$

The terms $p_{f_{p}}(f), p_{f_{m_{1}}^{T}}(f)$, and $p_{f_{m_{2}}^{T}}(f)$ stand for the PDF of $f_{p}, f_{m_{1}}^{T}$, and $f_{m_{2}}^{T}$, respectively. The AOD $\alpha_{p}$ and the Doppler frequency $f_{p}$ are related according to (10b), whereas the PDF of the AOD $\alpha_{p}$ is 
given by (16). Using the concept of transformation of RVs, the PDF of $f_{p}$ can be obtained as

$$
p_{f_{p}}(f)= \begin{cases}\frac{2}{\Delta \gamma_{p}} \frac{h_{T}^{1}\left(\arccos \left(\frac{f}{f_{\max }}\right)\right)+h_{T}^{1}\left(-\arccos \left(\frac{f}{f_{\max }}\right)\right)}{\sqrt{f_{\max }^{2}-f^{2}}} & \text { if } \cos \left(\alpha_{0}^{\text {out }}-\phi_{T}\right) \leq \frac{f}{f_{\max }} \leq 1 \\ \frac{2}{\Delta \gamma_{p}} \frac{h_{T}^{1}\left(\arccos \left(\frac{f}{f_{\max }}\right)\right)}{\sqrt{f_{\max }^{2}-f^{2}}} & \text { if } \cos \left(\alpha_{1}^{\text {out }}-\phi_{T}\right) \leq \frac{f}{f_{\max }}<\cos \left(\alpha_{0}^{\text {out }}-\phi_{T}\right) \\ 0 & \text { otherwise, }\end{cases}
$$

where

$$
h_{T}^{1}(x)=\frac{1}{1+\frac{R_{2}^{2}-R_{T}^{2}}{h_{1}\left(x, R_{2}\right)}}
$$

and

$$
h_{1}\left(x, R_{2}\right)=R_{2}^{2}+R_{T}^{2}-2 R_{T} R_{2} \cdot \cos \left(\arcsin \left(\frac{R_{T} \cdot \sin \left(\frac{\pi}{2}-x\right)}{R_{2}}\right)-\frac{\pi}{2}+x\right) .
$$

Analogously, we can find an expression for the PDF of $f_{m_{2}}^{T}$ as

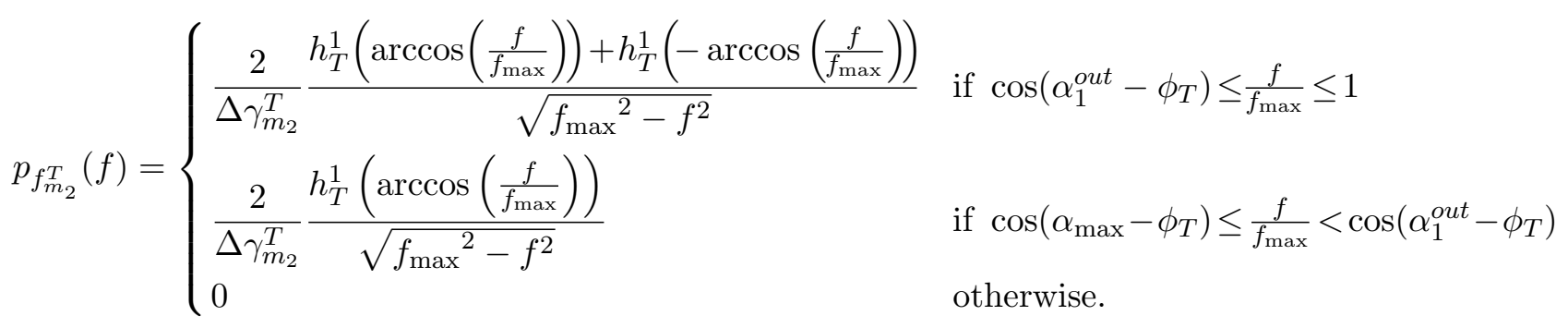

Similarly, we can derive an expression for the PDF of $f_{m_{1}}^{T}$ as

$$
p_{f_{m_{1}}^{T}}(f)= \begin{cases}\frac{2}{\Delta \gamma_{m_{1}}^{T}} \frac{h_{T}^{2}\left(\arccos \left(\frac{f}{f_{\max }}\right)\right)+h_{T}^{2}\left(-\arccos \left(\frac{f}{f_{\max }}\right)\right)}{\sqrt{f_{\max }^{2}-f^{2}}} & \text { if } \cos \left(\alpha_{1}-\phi_{T}\right) \leq \frac{f}{f_{\max }} \leq 1 \\ \frac{2}{\Delta \gamma_{m_{1}}^{T}} \frac{h_{T}^{2}\left(\arccos \left(\frac{f}{f_{\max }}\right)\right)}{\sqrt{f_{\max }^{2}-f^{2}}} & \text { if } \cos \left(\alpha_{\min }-\phi_{T}\right) \leq \frac{f}{f_{\max }}<\cos \left(\alpha_{1}-\phi_{T}\right) \\ 0 & \text { otherwise, }\end{cases}
$$

where

$$
h_{T}^{2}(x)=\frac{1}{1+\frac{R_{1}^{2}-R_{T}^{2}}{h_{2}\left(x, R_{1}\right)}}
$$

and

$$
h_{2}\left(x, R_{1}\right)=R_{1}^{2}+R_{T}^{2}-2 R_{T} R_{1} \cdot \cos \left(\arcsin \left(\frac{R_{T} \cdot \sin \left(\frac{\pi}{2}-x\right)}{R_{1}}\right)-\frac{\pi}{2}+x\right)
$$




\subsection{Numerical and Simulation Results}

In this section, the statistical properties of the V2I channel presented in the previous section are evaluated numerically and illustrated. The validity of the analytical expressions of these statistical properties is confirmed by simulations. The simulation model is designed using the sum-of-cisoids method [16] which is suitable for non-isotropic scattering environments as it is the case for the blind bend model. The simulation model parameters are computed using the modified method of equal areas (MMEA) proposed in [17]. For the reference model, the parameters have been chosen as follows: $R_{1}=6 \mathrm{~m}, R_{2}=12 \mathrm{~m}, R_{T}=7 \mathrm{~m}, R_{R}=11 \mathrm{~m}, \gamma_{R}=\pi / 7$, and $f_{\max }=91 \mathrm{~Hz}$. For the mean powers of the $\mathrm{SB}$ and the $\mathrm{DB}$ components, we have selected the values $\eta_{\mathrm{SB}}=0.5, \eta_{\mathrm{DBT}}=0.3$, and $\eta_{\mathrm{DBR}}=0.2$. Note that the total mean power of the channel is normalized to unity since $\eta_{\mathrm{SB}}+\eta_{\mathrm{DBT}}+\eta_{\mathrm{DBR}}=1$.

In Fig. 3, we illustrate the PDF of the AOD $\alpha_{p}$ for different values of $\gamma_{T}$. In the same figure, the analytical expression of the $\mathrm{PDF} p_{\alpha_{p}}\left(\alpha_{p}\right)$ is validated by simulation. We notice that as the transmitter MS moves towards the receiver RSU, the angular spread of the AOD $\alpha_{p}$ increases. Note that as $\gamma_{T}$ decreases the transmitter approaches the receiver. In Fig. 3, the simulation results for the PDF of the AOD have been obtained as follows. First, the AOS $\gamma_{p}$ are generated. The AOS are uniformly distributed in the interval $\left[\gamma_{0}^{\text {out }}, \gamma_{1}^{\text {out }}\right]$. Using the relation between the AOD $\alpha_{p}$ and the AOS $\gamma_{p}$ in (4), we can obtain the AOD samples. Afterwards, we compute the histogram of the generated AOD. Finally, the PDF of the AOD $\alpha_{p}$ can be determined from the histogram. Due to space limitation, the PDF of the AODs $\alpha_{m_{2}}^{T}$ and $\alpha_{m_{1}}^{T}$ have not been included. It is worth mentioning that the study of the PDF of $\alpha_{p}$ is more important since it has a larger impact on the channel statistics. Actually, the channel gains $g^{\mathrm{SB}}(t)$ and $g^{\mathrm{DBR}}(t)$ depend only of the AOD $\alpha_{p}$. The mean power associated with these components represents $70 \%$ of the total mean power of the channel.

The absolute value of the temporal ACF $|R(\tau)|$ is shown in Fig. 4. A good fitting can be observed between the temporal ACF of the reference and the simulation model, which confirms the correctness of the obtained results. From Fig. 4 we notice that the channel coherence time decreases as the transmitter moves towards the receiver. This can be explained by the fact that the angular spread increases as the transmitter approach the reviver (this fact is observed in Fig. 3). It is well known that the coherence time decreases as the angular spread increases. A short coherence time indicates the rapid variation of the channel, while a large coherence time implies that the channel varies slowly.

In Fig. 5, we depict the Doppler PSD $S_{g}(f)$ of the blind bend model for different values of $\gamma_{T}$. The theoretical results for the Doppler PSD are validated by simulations. The asymmetric shape of the Doppler PSD shows that we experience non-isotropic scattering conditions in blind bend environments.

In Fig. 6, we illustrate the impact of the radius $R_{2}$ on the PDF of the AOD and the temporal $\mathrm{ACF}|R(\tau)|$. The results in this figure have been obtained for the following simulation parameters: $R_{1}=6 \mathrm{~m}, R_{T}=7 \mathrm{~m}, \gamma_{R}=\pi / 7$, and $\gamma_{T}=11 \pi / 12$. It can be observed from Fig. 6 (a) that the angular spread of the $\mathrm{AOD} \alpha_{p}$ decays as $R_{2}$ decreases. Moreover, we notice that the range of interval $\left[\gamma_{0}^{\text {out }}, \gamma_{1}^{\text {out }}\right]$

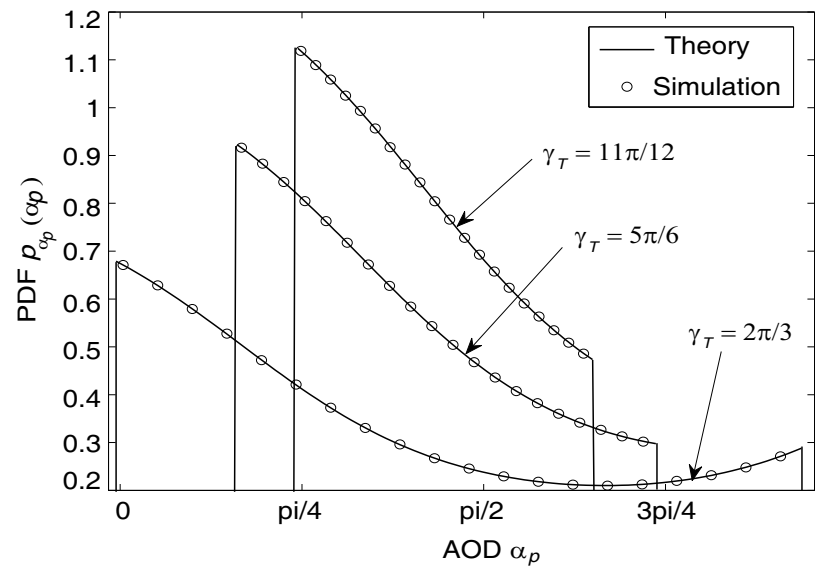

Figure 3. PDF of the AOD $\alpha_{p}$ for different values of $\gamma_{T}$.

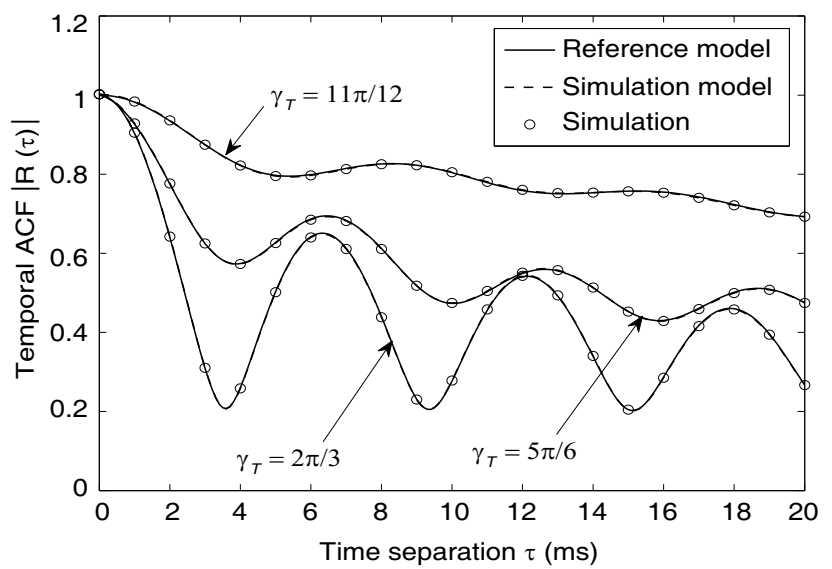

Figure 4. Absolute value of the temporal $|R(\tau)|$ of the blind bend model for different values of $\gamma_{T}$. 


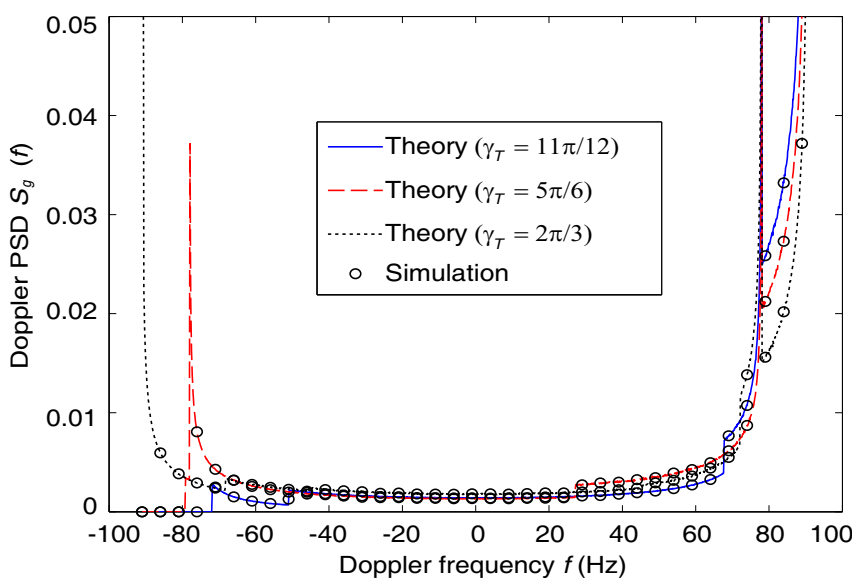

Figure 5. Doppler PSD $S_{g}(f)$ of the blind bend model for different values of $\gamma_{T}$.

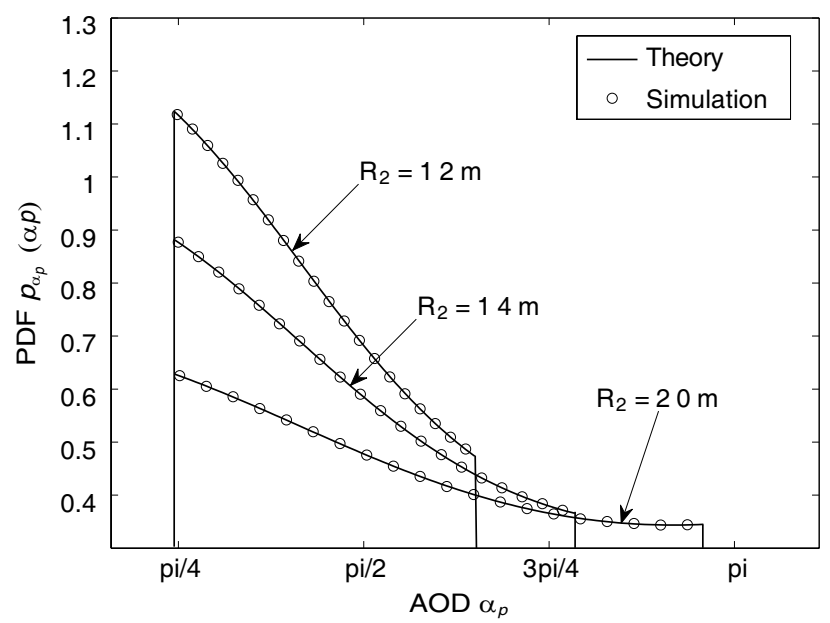

(a)

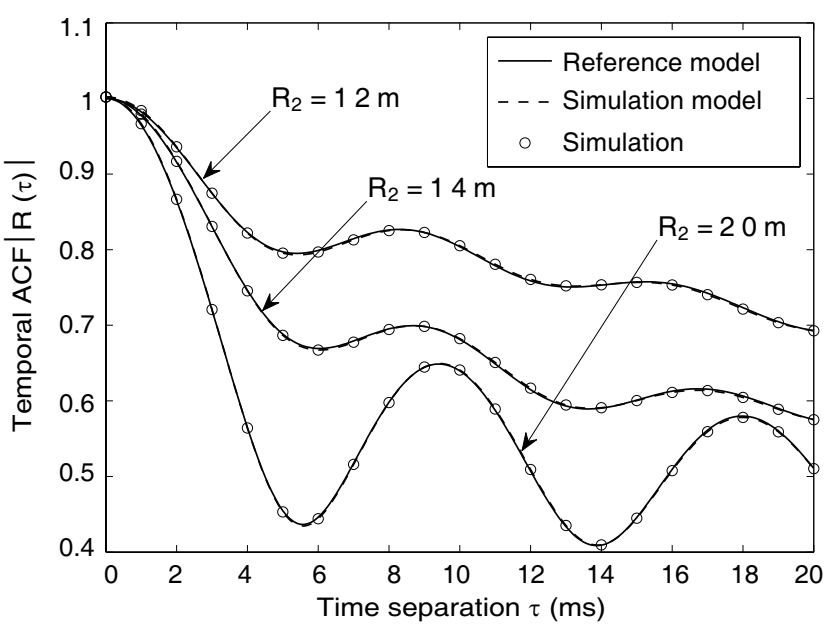

(b)

Figure 6. (a) PDF of the AOD $\alpha_{p}$ and (b) absolute value of the temporal $|R(\tau)|$ of the blind bend model for different values of $R_{2}$.

increases as $R_{2}$ increases, while the intervals $\left[\gamma_{1}^{\text {out }}, \gamma_{\max }\right]$ and $\left[\gamma_{\min }, \gamma_{0}^{\text {out }}\right]$ shrink. Note that the scatterers contributing to DB scattering are confined in the intervals $\left[\gamma_{1}^{\text {out }}, \gamma_{\max }\right]$ and $\left[\gamma_{\min }, \gamma_{0}^{\text {out }}\right]$. Therefore, as the radius $R_{2}$ becomes large the contribution of the DB component becomes very small, whereas the contribution of SB scattering increases. Additionally, we evaluate the impact of the radius $R_{2}$ on the temporal ACF in Fig. 6(b). It can be seen there that coherence time decreases as $R_{2}$ increases. This can be explained by the fact that the angular spread increases with the radius as shown in Fig. 6(a). It is an established fact the coherence time decreases as the angular spread increases.

\section{PERFORMANCE ANALYSIS}

The proposed V2I channel model takes into consideration SB and DB scattering. Under such conditions, the investigated channel falls under the umbrella of SSDS channels. For vehicular environments, measurements results in $[9,10]$ have confirmed that models that take into account SB and DB components are quite accurate and can emulate the fading channel behaviour with a great precision. In this section, we investigate some characteristic quantities of the SSDS channel. In particular, we derive analytical expressions for the PDF of the SNR, the outage probability, and the MGF of the SNR. 
Moreover, we determine the expressions of the PDF and the cumulative distribution function (CDF) of the capacity, as well as the outage capacity. Additionally, analytical expressions are obtained for the average SEP of M-PSK and M-QAM modulation schemes without diversity, with MRC combining, and with SC combining. The validity of the theoretical results is confirmed by Monte Carlo simulations.

\subsection{Characteristic Quantities of SSDS Channels}

In Section 3, we provide in (8) an expression for the channel gain describing the link between the transmitter and the receiver for the proposed V2I channel model. This channel gain can be rewritten in the following form

$$
g(t)=g^{\mathrm{SB}}(t)+g^{\mathrm{DB}}(t)=w_{1} g_{1}(t)+w_{2} g_{2}(t) g_{3}(t)
$$

The first term in (34) is associated with SB scattering, while the second term is caused by DB scattering. The processes $g_{i}(t)$ are i.i.d. complex Gaussian processes with zero mean and unit variance. The amplitudes $\left|g_{1}(t)\right|$ and $\left|g_{2}(t) g_{3}(t)\right|$ follow a Rayleigh and a double Rayleigh distribution, respectively. The weighting factors $w_{1}$ and $w_{2}$ determine the contributions of the SB and the DB component. Henceforth, we drop the time dependence for ease of notation. We denote by $G$ the amplitude of the channel gain, i.e., $G=|g|$. For SSDS channels, the PDF of the amplitude of the channel gain $G$ can be expressed in series form as $[8]$

$$
p_{G}(r)=\sum_{n=0}^{\infty} a_{n} r^{2 n+1}
$$

where

$$
a_{n}=2 e^{w_{1}^{2} / w_{2}^{2}} \frac{(-1)^{n}}{n !} \frac{\Gamma_{u}\left(-n, \frac{w_{1}^{2}}{w_{2}^{2}}\right)}{\left(w_{2}^{2}\right)^{n+1}} .
$$

It has to be mentioned that in $(36) \Gamma_{u}(\cdot, \cdot)$ stands for the upper incomplete gamma function given by $\Gamma_{u}(a, x)=\int_{x}^{\infty} t^{a-1} e^{-t} d t$.

Next, we determine the analytical expressions of the PDF of the SNR, the outage probability, and the MGF of the SNR. The SNR $\gamma$ is defined as

$$
\gamma=\frac{E_{s}}{N_{0}} G^{2}=\rho_{s} G^{2}
$$

The term $E_{s}$ denotes the symbol energy and $N_{0}$ stands for the noise power. Using the theorem of transformation of random variables, we can obtain the PDF of $\gamma$ from the PDF of $G$ as

$$
p_{\gamma}(\gamma)=\frac{1}{2 \rho_{s}} \sum_{n=0}^{\infty} a_{n}\left(\frac{\gamma}{\rho_{s}}\right)^{n}
$$

The outage probability is defined as the probability that the SNR drops below a threshold level $\gamma_{\text {th }}$. This probability can be evaluated using the CDF of $\gamma$ and can be written as

$$
P_{\text {out }}=\mathbb{P}\left\{\gamma<\gamma_{\mathrm{th}}\right\}=\frac{1}{2 \rho_{s}} \sum_{n=0}^{\infty} \frac{a_{n}}{(n+1) \rho_{s}^{n}} \gamma_{\mathrm{th}}{ }^{n+1} .
$$

The MGF of the SNR $\gamma$ is defined as

$$
M_{\gamma}(s)=\int_{0}^{\infty} \exp (\gamma s) p_{\gamma}(\gamma) d \gamma=\sum_{n=0}^{\infty} \frac{(-1)^{n+1} a_{n} n !}{2\left(s \rho_{s}\right)^{n+1}}
$$


In the following, we derive closed-form expressions for the PDF and the CDF of the capacity of SSDS channels in series form. The normalized channel capacity is equal to $C=\log _{2}(1+\gamma)$ [18]. The $\mathrm{PDF} p_{C}(r)$ of the channel capacity is obtained using $p_{C}(r)=2^{r} \ln (2) p_{\gamma}\left(2^{r}-1\right)$ as follows

$$
p_{C}(r)=\frac{\ln (2) 2^{r}}{2 \rho_{s}} \sum_{n=0}^{\infty} a_{n}\left(\frac{2^{r}-1}{\rho_{s}}\right)^{n} .
$$

To compute the CDF of the capacity, we make use of the identity $P_{C}(r)=\int_{0}^{r} p_{C}(x) d x$. Solving this integral, we can express this CDF as

$$
P_{C}(r)=\frac{1}{2} \sum_{n=0}^{\infty} \frac{a_{n}}{n+1}\left(\frac{2^{r}-1}{\rho_{s}}\right)^{n+1}
$$

Moreover, we determine an expression for the outage capacity. This quantity is defined as the probability that the channel capacity falls below a rate $R$. The outage capacity can be written as

$$
C_{\text {out }}=\mathbb{P}\left\{\log _{2}(1+\gamma)<R\right\}=P_{C}(R)=\frac{1}{2} \sum_{n=0}^{\infty} \frac{a_{n}}{n+1}\left(\frac{2^{R}-1}{\rho_{s}}\right)^{n+1} .
$$

\subsection{Performance Analysis in SSDS Channels without Diversity}

This section is devoted to study the performance of M-PSK and M-QAM modulation schemes over SSDS channels without diversity. In the case of transmission over a fading channel, the SEP $P_{s}$ is obtained by averaging the conditional SEP $P_{e \mid \gamma}$ over the fading statistics as [19]

$$
P_{s}=\int_{0}^{\infty} P_{e \mid \gamma} p_{\gamma}(\gamma) d \gamma
$$

The conditional SEP $P_{e \mid \gamma}$ for the M-PSK modulation scheme is given by [19, (Eq. (8.23)]

$$
P_{e \mid \gamma}=\frac{1}{\pi} \int_{0}^{(M-1) \pi / M} \exp \left(-\frac{\gamma g_{\mathrm{PSK}}}{\sin ^{2}(\theta)}\right) d \theta
$$

where $g_{\mathrm{PSK}}=\sin ^{2}(\pi / M)$. Substituting (45) in (44) and using [20, Eq. (1.5.2.3)], we can determine a closed-form expression for the average SEP of the M-PSK modulation scheme over an SSDS channel as

$$
\begin{aligned}
P_{s}= & \int_{0}^{\infty} \frac{1}{\pi} \int_{0}^{(M-1) \pi / M} \exp \left(-\frac{\gamma g_{\mathrm{PSK}}}{\sin ^{2}(\theta)}\right) p_{\gamma}(\gamma) d \gamma d \theta \\
= & \frac{1}{\pi} \int_{0}^{(M-1) \pi / M} M_{\gamma}\left(-\frac{g_{\mathrm{PSK}}}{\sin ^{2}(\theta)}\right) d \theta \\
= & \frac{1}{\pi} \sum_{n=0}^{\infty} \frac{a_{n} n !}{2\left(\rho_{s} g_{\mathrm{PSK}}\right)^{n+1}}\left[\frac{1}{2^{2 n+2}}\left(\begin{array}{c}
2 n+2 \\
n+1
\end{array}\right) \frac{(M-1) \pi}{M}\right. \\
& \left.+\frac{(-1)^{n+1}}{2^{2 n+1}} \sum_{k=0}^{n}(-1)^{k}\left(\begin{array}{c}
2 n+2 \\
k
\end{array}\right) \frac{\sin \left((n+1-k) \frac{(M-1) 2 \pi}{M}\right)}{2 n+2-2 k}\right] .
\end{aligned}
$$

For an M-QAM modulation scheme, the conditional SEP $P_{e \mid \gamma}$ reads as [19, Eq. (8.12)]

$$
P_{e \mid \gamma}=\frac{4 \alpha}{\pi} \int_{0}^{\pi / 2} \exp \left(-\frac{\gamma \beta}{2 \sin ^{2}(\theta)}\right) d \theta-\frac{4 \alpha^{2}}{\pi} \int_{0}^{\pi / 4} \exp \left(-\frac{\gamma \beta}{2 \sin ^{2}(\theta)}\right) d \theta
$$


where $\alpha=\frac{\sqrt{M}-1}{\sqrt{M}}$ and $\beta=\frac{3}{M-1}$. Replacing (47) in (44) and using an MGF-based approach [19], we can obtain a closed-form expression for the average SEP of M-QAM modulation scheme over an SSDS channel as

$$
\begin{aligned}
P_{s}= & \frac{4 \alpha}{\pi} \int_{0}^{\pi / 2} M_{\gamma}\left(-\frac{\beta}{2 \sin ^{2}(\theta)}\right) d \theta-\frac{4 \alpha^{2}}{\pi} \int_{0}^{\pi / 4} M_{\gamma}\left(-\frac{\beta}{2 \sin ^{2}(\theta)}\right) d \theta \\
= & \frac{4 \alpha}{\pi} \sum_{0}^{\infty} \frac{a_{n} 2^{n} n !}{\left(\beta \rho_{s}\right)^{n+1}}\left[\frac{1}{2^{2 n+2}}\left(\begin{array}{c}
2 n+2 \\
n+1
\end{array}\right) \frac{\pi}{2}\right]-\frac{4 \alpha^{2}}{\pi} \sum_{0}^{\infty} \frac{a_{n} 2^{n} n !}{\left(\beta \rho_{s}\right)^{n+1}} \\
& {\left[\frac{1}{2^{2 n+2}} \cdot\left(\begin{array}{c}
2 n+2 \\
n+1
\end{array}\right) \frac{\pi}{4}+\frac{(-1)^{n+1}}{2^{2 n+1}} \sum_{k=0}^{n}(-1)^{k}\left(\begin{array}{c}
2 n+2 \\
k
\end{array}\right) \frac{\sin \left((n+1-k) \frac{\pi}{2}\right)}{2 n+2-2 k}\right] . }
\end{aligned}
$$

\subsection{Performance Analysis in SSDS Channels with MRC Combining}

In this section, we investigate the performance of M-PSK and M-QAM modulation schemes over SSDS channel with MRC combining. We consider an $L$-branch diversity system. Let $\gamma_{k}(k=1,2, \ldots, L)$ stand for the SNR associated with the $k$ th diversity branch. We utilize an MGF-based approach [21] to derive the expression of the average SEP. The MGF of the random variable (RV) $\gamma_{k}$ is given by (40). The SNR at the output of the MRC combiner is referred to as $\gamma_{T}$ and can be expressed as $\gamma_{T}=\sum_{k=1}^{L} \gamma_{k}$. The MGF of the RV $\gamma_{T}$ can be obtained as

$$
M_{\gamma_{T}}(s)=E\left(e^{s \sum_{k=1}^{L} \gamma_{k}}\right)=\left(\frac{1}{2} \sum_{n=0}^{\infty} \frac{a_{n}(-1)^{n+1} n !}{\left(s \rho_{s}\right)^{n+1}}\right)^{L} .
$$

Using the MGF-based approach [19], we can compute the average SEP for M-PSK with MRC combining as

$$
\begin{aligned}
P_{s}= & \frac{1}{\pi} \int_{0}^{(M-1) \pi / M} M_{\gamma_{T}}\left(-\frac{g_{\mathrm{PSK}}}{\sin ^{2}(\theta)}\right) d \theta=\frac{1}{\pi} \int_{0}^{(M-1) \pi / M} \sin (\theta)^{2 L}\left(\sum_{n=0}^{\infty} b_{n} \sin (\theta)^{2 n}\right)^{L} d \theta \\
= & \frac{1}{\pi} \int_{0}^{(M-1) \pi / M} \sum_{n=0}^{\infty} c_{n} \sin (\theta)^{2(n+L)} d \theta=\frac{1}{\pi} \sum_{n=0}^{\infty} c_{n}\left[\frac{1}{2^{2(n+L)}}\left(\begin{array}{c}
2(n+L) \\
n+L
\end{array}\right) \frac{(M-1) \pi}{M}\right. \\
& \left.+\frac{(-1)^{n+L}}{2^{2(n+L)-1}} \sum_{k=0}^{n+L-1}(-1)^{k}\left(\begin{array}{c}
2(n+L) \\
k
\end{array}\right) \frac{\sin \left(\frac{(2 n+2 L-2 k)(M-1) \pi}{M}\right)}{2 n+2 L-2 k}\right],
\end{aligned}
$$

where

$$
\begin{aligned}
b_{n} & =\frac{n ! a_{n}}{2\left(\rho_{s} g_{\mathrm{PSK}}\right)^{n+1}} \\
c_{0} & =b_{0}{ }^{L} \\
c_{k} & =\frac{1}{k b_{0}} \sum_{n=1}^{k} b_{n}(n L-k+n) c_{k-n} .
\end{aligned}
$$

For an M-QAM modulation scheme, the SEP of MRC combining with $L$-diversity branches can be 
determined as

$$
\left.\begin{array}{rl}
P_{s}= & \frac{4 \alpha}{\pi} \int_{0}^{\pi / 2} M_{\gamma_{T}}\left(-\frac{\beta}{2 \sin ^{2}(\theta)}\right) d \theta-\frac{4 \alpha^{2}}{\pi} \int_{0}^{\pi / 4} M_{\gamma_{T}}\left(-\frac{\beta}{2 \sin ^{2}(\theta)}\right) d \theta \\
= & \frac{4 \alpha}{\pi} \int_{0}^{\pi / 2} \sin (\theta)^{2 L}\left(\sum_{n=0}^{\infty} x_{n} \sin (\theta)^{2 n}\right)^{L} d \theta-\frac{4 \alpha^{2}}{\pi} \int_{0}^{\pi / 4} \sin (\theta)^{2 L}\left(\sum_{n=0}^{\infty} x_{n} \sin (\theta)^{2 n}\right)^{L} d \theta \\
= & \frac{4}{\pi} \sum_{n=0}^{\infty} y_{n}\left[\alpha \int_{0}^{\pi / 2} \sin (\theta)^{2(n+L)} d \theta-\alpha^{2} \int_{0}^{\pi / 4} \sin (\theta)^{2(n+L)} d \theta\right] \\
= & \frac{4}{\pi} \sum_{n=0}^{\infty} y_{n}\left[\alpha\left[\frac{1}{2^{2(n+L)}}\left(\begin{array}{c}
2(n+L) \\
n+L
\end{array}\right) \frac{\pi}{2}+\frac{(-1)^{n+L}}{2^{2(n+L)-1}} \sum_{k=0}^{n+L-1}(-1)^{k} \cdot\left(\begin{array}{c}
2(n+L) \\
k
\end{array}\right) \frac{\sin \left(\frac{(2 n+2 L-2 k) \pi}{2}\right)}{2 n+2 L-2 k}\right]\right. \\
& -\alpha^{2}\left[\frac{1}{2^{2(n+L)}}\left(\begin{array}{c}
2(n+L) \\
n+L
\end{array}\right) \frac{\pi}{4}+\frac{(-1)^{n+L}}{2^{2(n+L)-1}} \sum_{k=0}^{n+L-1}(-1)^{k}\left(\begin{array}{c}
2(n+L) \\
k
\end{array}\right) \frac{\left.\sin \left(\frac{(2 n+2 L-2 k) \pi}{4}\right)\right]}{2 n+2 L-2 k}\right]
\end{array}\right]
$$

with

$$
\begin{aligned}
x_{n} & =\frac{2^{n} n ! a_{n}}{\left(\beta \rho_{s}\right)^{n+1}} \\
y_{0} & =x_{0}{ }^{L} \\
y_{k} & =\frac{1}{k x_{0}} \sum_{n=1}^{k} x_{n}(n L-k+n) y_{k-n} .
\end{aligned}
$$

\subsection{Performance Analysis in SSDS Channels with SC Combining}

In this section, our investigations focus on the performance of different modulation schemes with SC combining. We derive closed-form expressions for the SEP of M-PSK and M-QAM modulation schemes for the case of SC diversity combining over SSDS channels. Towards this aim, we first need to determine the PDF of the SNR $\gamma_{s}$ at the output of the SC diversity combiner. The SNR $\gamma_{s}$ can be written as

$$
\gamma_{s}=\max \left(\gamma_{1}, \gamma_{2}, \ldots, \gamma_{L}\right)
$$

The CDF of the RV $\gamma_{s}$ can be obtained as

$$
P_{\gamma_{s}}(x)=\mathbb{P}\left\{\gamma_{s} \leq x\right\}=\left(\frac{1}{2} \sum_{n=0}^{\infty} \frac{a_{n} x^{n+1}}{(n+1) \rho_{s}^{n+1}}\right)^{L} .
$$

It follows that the PDF of $\gamma_{s}$ can be expressed as

$$
\begin{aligned}
p_{\gamma_{s}}(x) & =\frac{d P_{\gamma_{s}}(x)}{d x}=L\left(\frac{1}{2} \sum_{n=0}^{\infty} \frac{a_{n}}{(n+1) \rho_{s}{ }^{n+1}} x^{n+1}\right)^{L-1}\left(\frac{1}{2} \sum_{n=0}^{\infty} \frac{a_{n}}{\rho_{s}{ }^{n+1}} x^{n}\right) \\
& =L x^{L-1}\left(\sum_{n=0}^{\infty} d_{n} x^{n}\right)^{L-1}\left(\sum_{n=0}^{\infty} e_{n} x^{n}\right)=L x^{L-1}\left(\sum_{n=0}^{\infty} f_{n} x^{n}\right)\left(\sum_{n=0}^{\infty} e_{n} x^{n}\right)
\end{aligned}
$$


where

$$
\begin{aligned}
e_{n} & =\frac{a_{n}}{2 \rho_{s}{ }^{n+1}} \\
d_{n} & =\frac{a_{n}}{2(n+1) \rho_{s}{ }^{n+1}} \\
f_{0} & =\left(d_{0}\right)^{L-1} \\
f_{n} & =\frac{1}{n d_{0}} \sum_{m=1}^{n}(m(L-1)-n+m) d_{m} f_{m-n} .
\end{aligned}
$$

Using [22, Eq. (0.316)], we can further simplify the expression of this PDF as

$$
p_{\gamma_{s}}(x)=L \sum_{n=0}^{\infty} h_{n} x^{n+L-1},
$$

with $h_{n}=\sum_{k=0}^{n} f_{k} e_{n-k}$. Thus, we can get a closed-form result for the SEP of M-PSK with SC diversity combining as

$$
\begin{aligned}
P_{s}= & \int_{0}^{\infty} P_{e \mid \gamma_{s}} p_{\gamma_{s}}(x) d x=\frac{1}{\pi} \int_{0}^{\infty} \int_{0}^{(M-1) \pi / M} \sum_{n=0}^{\infty} L h_{n} x^{n+L-1} \exp \left(-\frac{x g_{\mathrm{PSK}}}{\sin (\theta)^{2}}\right) d \theta d x \\
= & \frac{1}{\pi} \sum_{n=0}^{\infty} L h_{n} \int_{0}^{(M-1) \pi / M}\left(\frac{\sin (\theta)^{2}}{g_{\mathrm{PSK}}}\right)^{n+L}(n+L-1) ! d \theta \\
= & \sum_{n=0}^{\infty} \frac{L h_{n}(n+L-1) !}{\pi\left(g_{\mathrm{PSK}}\right)^{n+L}}\left[\frac{1}{2^{2(n+L)}\left(\begin{array}{c}
2(n+L) \\
n+L
\end{array}\right) \frac{(M-1) \pi}{M}}\right) \\
& \left.+\frac{(-1)^{n+L}}{2^{2(n+L)-1}} \sum_{k=0}^{n+L-1}(-1)^{k}\left(\begin{array}{c}
2(n+L) \\
k
\end{array}\right) \frac{\sin \left(\frac{2(n+L-k)(M-1) \pi}{M}\right)}{2(n+L-k)}\right]
\end{aligned}
$$

For M-QAM modulation scheme, the average SEP of SC diversity combining can be determined as

$$
\begin{aligned}
P_{s}= & \int_{0}^{\infty} P_{e \mid \gamma_{s}} p_{\gamma_{s}}(x) d x=\sum_{n=0}^{\infty} \frac{4 L h_{n}}{\pi}\left[\alpha \int_{0}^{\infty} \int_{0}^{\pi / 2} x^{n+L-1} \exp \left(-\frac{\beta x}{2 \sin (\theta)^{2}}\right) d \theta d x\right. \\
& \left.-\alpha^{2} \int_{0}^{\infty} \int_{0}^{\pi / 4} x^{n+L-1} \exp \left(-\frac{\beta x}{2 \sin (\theta)^{2}}\right) d \theta d x\right] \\
= & \sum_{n=0}^{\infty} \frac{4 L h_{n}(n+L-1) !}{\pi \beta^{n+L}} 2^{n+L}\left[\alpha \int_{0}^{\pi / 2}(\sin (\theta))^{2(n+L)} d \theta-\alpha^{2} \int_{0}^{\pi / 4}(\sin (\theta))^{2(n+L)} d \theta\right] \\
= & \sum_{n=0}^{\infty} \frac{4 L h_{n}(n+L-1) !}{\pi \beta^{n+L}} 2^{n+L}\left[\alpha\left[\frac{1}{2^{2(n+L)}}\left(\begin{array}{c}
2(n+L) \\
n+L
\end{array}\right) \frac{\pi}{2}\right]\right. \\
& -\alpha^{2}\left[\frac{1}{2^{2(n+L)}}\left(\begin{array}{c}
2(n+L) \\
n+L
\end{array}\right) \frac{\pi}{4}+\frac{(-1)^{n+L}}{2^{2(n+L)-1}} \sum_{k=0}^{n+L-1}(-1)^{k}\left(\begin{array}{c}
2(n+L) \\
k
\end{array}\right) \frac{\left.\sin \left(\frac{(2 n+2 L-2 k) \pi}{2 n+2 L-2 k}\right)\right]}{4}\right] .
\end{aligned}
$$




\subsection{Numerical and Simulation Results}

In this section, we evaluate numerically the analytical expressions of the SEP derived in the previous sections. We also confirm the validity of the obtained theoretical results using Monte Carlo simulations. In Fig. 7, we depict the SEP of BPSK modulation scheme over an SSDS channel and a Rayleigh channel. From this figure, we can clearly see that the SEP is larger in the case of SSDS channels. Thus, the severity of fading of SSDS channels is higher compared to Rayleigh channels. This fact motivates us to investigate the performance of different kind of modulations schemes over SSDS channels. The results in Fig. 7 have been obtained for the case of MRC combining with three diversity branches. The parameters $w_{1}$ and $w_{2}$ characterizing the power of the SB component and the DB component have been set to 1 and $\sqrt{2}$, respectively. From Fig. 7 , we can observe a degradation of $1.5 \mathrm{~dB}$ at an SEP of $10^{-3}$ for the SSDS channel compared to the Rayleigh channel. This degradation increases as the ratio $\left(w_{2}^{2} / w_{1}^{2}\right)$ increases. A maximum channel severity is reached when $w_{1}$ is equal to zero, while $w_{2}$ is nonzero. Under such conditions, we have pure DB scattering and the fading amplitude follow a double Rayleigh distribution. The generality of the SSDS channel drives from the fact that it incorporates the Rayleigh and the double Rayleigh channel as special cases. A transmitted signal experiences in general a combination of SB and DB scattering making the SSDS channel appropriate for describing the fading amplitude of real-world

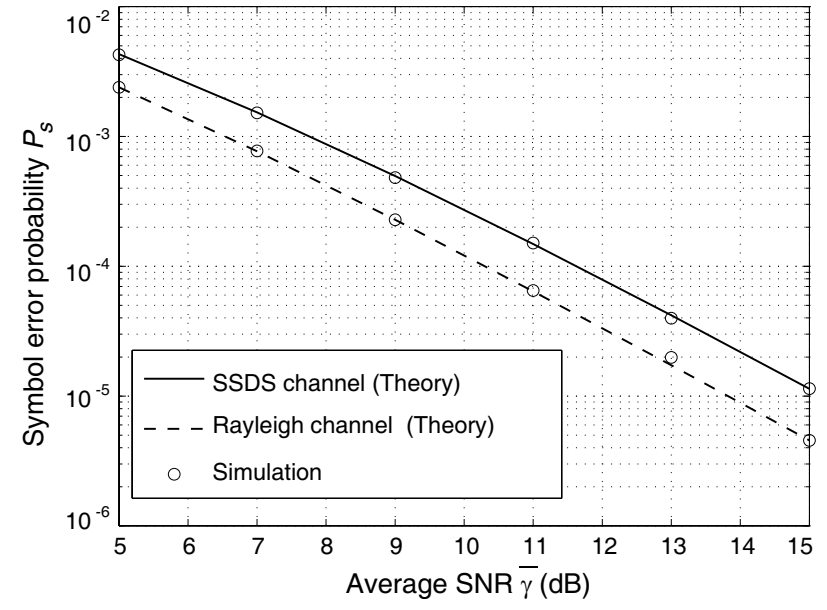

Figure 7. SEP of BPSK with MRC combining over Rayleigh and SSDS channels.

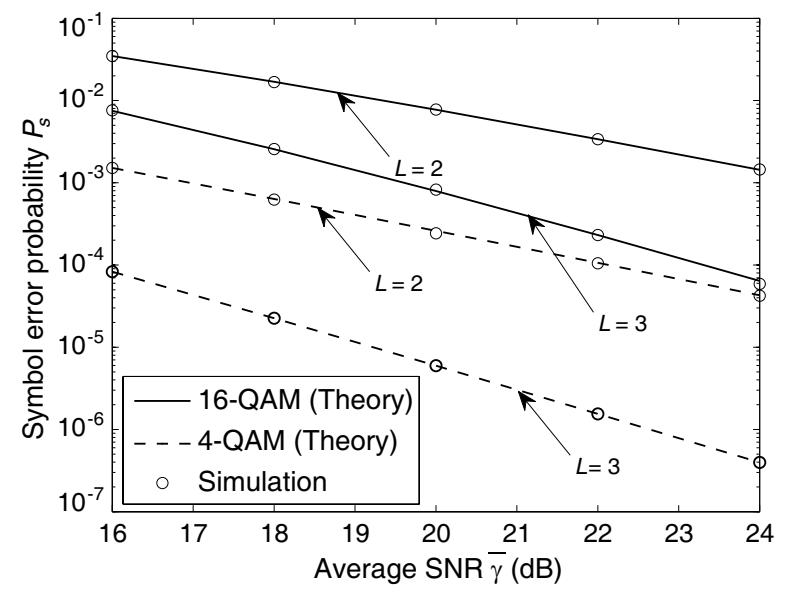

Figure 9. SEP of M-QAM with MRC combining for different numbers $L$ of diversity branches.

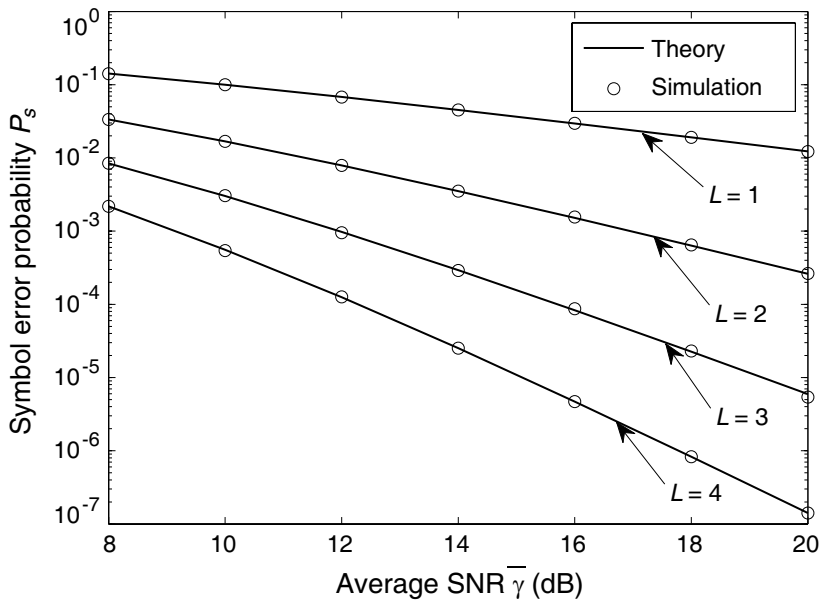

Figure 8. SEP of QPSK with MRC combining for different numbers $L$ of diversity branches.

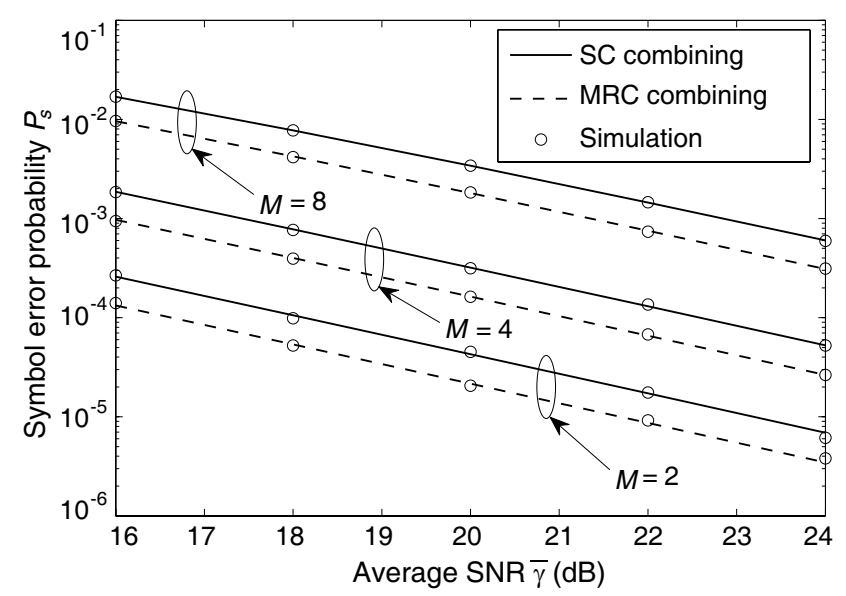

Figure 10. SEP of M-PSK with SC and MRC combining over SSDS channels. 
propagation channels. The contribution of the SB component and the DB component differs depending on the propagation scenario. The SSDS channel is well suited for describing the fading amplitude for V2I propagation scenarios. Hence, for developing future V2I communication systems a deep investigation of different modulation schemes over SSDS channel is required.

In Fig. 8, we evaluate the SEP of QPSK with MRC combining. From this figure, we can clearly see that the SEP decreases as the number of diversity branches, $L$, increases. In Fig. 9, we present the SEP of 4-QAM and 16-QAM modulation schemes. These results have been obtained for the case of MRC combining. The number of diversity branches has been set to two and three. From this figure, we can conclude that the SEP increases as the constellation size increases. For a 16-QAM modulation scheme, we achieve a gain of $4 \mathrm{~dB}$ at an SEP of $10^{-2}$ by increasing the number of diversity branches from two to three. For 4-QAM modulation scheme, at an SEP of $10^{-4}$ a $6 \mathrm{~dB}$ gain is achieved if we use three receiving antennas instead of two. It has to be noted that the expressions of the SEP presented in the previous sections are in series form. The convergence region of these series depends on the type of modulation scheme as well as the diversity combining technique used. For instance, for QPSK with MRC combining, the SEP given by (50) converges for an average SNR $\bar{\gamma}$ larger than $8 \mathrm{~dB}$.

We illustrate in Fig. 10 the SEP of BPSK, 4-PSK, and 8-PSK in the case of SC and MRC combining with two diversity branches. The parameters $w_{1}$ and $w_{2}$ of the SSDS channel have been set to $\sqrt{2}$ and 1, respectively. From Fig. 10, it can be seen that the MRC combining scheme performs better than the SC combining scheme. For instance, at an SEP of $10^{-3}$, the use of MRC diversity technique allows achieving a $1.5 \mathrm{~dB}$ gain compared to the SC scheme in the case of 4-PSK modulation. Additionally, it can be observed that the SEP increases as the constellation size $M$ increases. For SC combining and at an SEP of $10^{-3}$, a degradation of $5.5 \mathrm{~dB}$ occurs if we use 8-QAM modulation instead of 4-QAM modulation.

\section{CONCLUSION}

In this paper, we introduced a novel SISO V2I channel model for blind bend environments. The proposed model takes into consideration SB and DB scattering components. The statistical properties of the proposed blind bend model have been investigated. Analytical expressions for the PDF of the AOD, the temporal ACF, and the Doppler PSD have been derived. The proposed model is fairly flexible since the street parameters can be modified to fit various curved streets with different layouts and dimensions. In this paper, we studied the impact of the position of the transmitter on the channel statistics. Supported by our analysis, we can conclude that as the transmitter approaches the receiver the angular spread increases and the channel coherence time decreases.

For the proposed V2I channel model, the transmitted waves experience SB and DB scattering which make the channel SSDS. For such a channel, we showed that the severity of fading is higher compared to Rayleigh channels. In this work, we derived analytical expressions for some characteristic quantities of the SSDS channel, such as the PDF and the MGF of the SNR as well as the PDF and the CDF of the capacity. Moreover, we provided analytical expressions for the SEP of M-PSK and M-QAM modulation schemes over SSDS fading channels without diversity, with MRC combining, and with SC combining. These results have been derived using an MGF-based approach. The validity of the obtained analytical expressions has been confirmed by Monte Carlo simulations. The results presented in this paper are useful for the development, design, and test of future V2I communication systems.

\section{REFERENCES}

1. Damoss, E. and L. M. Correia, "Digital mobile radio toward future generation systems (COST 231 Final Report)," Tech. Rep., European Communities, Brussels, Belgium, 1999.

2. Belanovic, P., D. Valerio, A. Paier, T. Zemen, F. Ricciato, and C. Mecklenbräuker, "On wireless links for vehicle-to-infrastructure communications," IEEE Transactions on Vehicular Technology, Vol. 59, No. 1, 269-282, Jan. 2010.

3. Gozalvez, J., M. Sepulcre, and R. Bauza, "IEEE 802.11p vehicle to infrastructure communications in urban environments," IEEE Communications Magazine, Vol. 50, No. 5, 176-183, May 2012. 
4. Shivaldova, V., G. Maier, D. Smely, N. Czink, A. Alonso, A. Winkelbauer, A. Paier, and C. Mecklenbräuker, "Performance evaluation of IEEE 802.11p infrastructure-to-vehicle tunnel measurements," 7th International Wireless Communications and Mobile Computing Conference (IWCMC 2011), 848-852, Istanbul, Turkey, Jul. 2011.

5. Alsehaili, M., S. Noghanian, A. R. Sebak, and D. A. Buchanan, "Angle and time of arrival statistics of a three dimensional geometrical scattering channel model for indoor and outdoor propagation environments," Progress In Electromagnetics Research, Vol. 109, 191-209, 2010.

6. Chen, Y., Z. Zhang, L. Hu, and P. Rapajic, "Geometry-based statistical model for radio propagation in rectangular office buildings," Progress In Electromagnetics Research B, Vol. 17, 187-212, 2009.

7. Chen, Y., Z. Zhang, and T. Qin, "Geometrically based channel model for indoor radio propagation with directional antennas," Progress In Electromagnetics Research B, Vol. 20, 109-124, 2010.

8. Salo, J., H. El-Sallabi, and P. Vainikainen, "Statistical analysis of the multiple scattering radio channel," IEEE Transactions on Antennas and Propagation, Vol. 54, No. 11, 3114-3124, Nov. 2006.

9. Zajić, A. G. and G. L. Stüber, "Three-dimensional modeling and simulation of wideband MIMO mobile-to-mobile channels," IEEE Transactions on Wireless Communications, Vol. 8, No. 3, 12601275, Mar. 2009.

10. Cheng, X., C.-X. Wang, D. Laurenson, S. Salous, and A. Vasilakos, "An adaptive geometrybased stochastic model for non-isotropic MIMO mobile-to-mobile channels," IEEE Transactions on Wireless Communications, Vol. 8, No. 9, 4824-4835, Sep. 2009.

11. Chelli, A., R. Hamdi, and M.-S. Alouini, "A vehicle-to-infrastructure channel model for blind corner scattering environments," Proc. IEEE 78th Vehicular Technology Conference (VTC2013-Fall), 1-6, Las Vegas, USA, Sep. 2013.

12. Chelli, A. and M. Pätzold, "The impact of fixed and moving scatterers on the statistics of MIMO vehicle-to-vehicle channels," Proc. IEEE 69th Vehicular Technology Conference (VTC2009-Spring), 1-6, Barcelona, Spain, Apr. 2009.

13. Zajić, A. G., G. L. Stüber, T. G. Pratt, and S. T. Nguyen, "Wideband MIMO mobile-to-mobile channels: geometry-based statistical modeling with experimental verification," IEEE Transactions on Vehicular Technology, Vol. 58, No. 2, 517-534, Feb. 2009.

14. Zhiyi, H., C. Wei, Z. Wei, M. Pätzold, and A. Chelli, "Modelling of MIMO vehicle-to-vehicle fading channels in T-junction scattering environments," Proc. 3rd European Conference on Antennas and Propagation (EuCAP 2009), 652-656, Berlin, Germany, Mar. 2009.

15. Papoulis, A. and S. U. Pillai, Probability, Random Variables and Stochastic Processes, 4th Edition, McGraw-Hill, New York, 2002.

16. Pätzold, M., Mobile Radio Channels, 2nd Edition, John Wiley \& Sons, Chichester, 2011.

17. Gutiérrez, C. A. and M. Pätzold, "Sum-of-sinosoids-based simulation of flat fading wireless propagation channels under non-isotropic scattering conditions," Proc. 50th IEEE Global Telecommunications Conference, (GLOBECOM 2007), 3842-3846, Washington DC, USA, Nov. 2007.

18. Winters, J., "On the capacity of radio communication systems with diversity in a Rayleigh fading environment," IEEE Journal on Selected Areas in Communications, Vol. 5, No. 5, 871878, Jun. 1987.

19. Simon, M. K. and M.-S. Alouini, Digital Communication over Fading Channels, 2nd edition, John Wiley \& Sons, New Jersey, USA, 2005.

20. Prudnikov, A., Y. Brychkov, and O. Marichev, Integrals and Series: Volume 1: Elementary Functions, Gordon and Breach Science Publishers, 1986.

21. Alouini, M.-S. and M. Simon, "An MGF-based performance analysis of generalized selection combining over Rayleigh fading channels," IEEE Transactions on Communications, Vol. 48, No. 3, 401-415, Mar. 2000.

22. Gradshteyn, I. S. and I. M. Ryzhik, Table of Integrals, Series, and Products, 7th Edtion, Academic Press, 2013. 